\title{
Activation of Infralimbic to Nucleus Accumbens Shell Pathway Suppresses Conditioned Aversion in Male But Not Female Rats
}

\author{
Seth W. Hurley and Regina M. Carelli \\ Department of Psychology and Neuroscience, The University of North Carolina at Chapel Hill, Chapel Hill, North Carolina 27599
}

\begin{abstract}
Hedonic processing plays an integral role in directing appropriate behavior, but disrupted hedonic processing is associated with psychiatric disorders such as depression. The infralimbic cortex (IL) is a key structure in affective processing in rodents and activation of its human homolog, the ventromedial prefrontal cortex, has been implicated in suppressing aversive states. Here, we tested whether optogenetic activation of glutamatergic projections from the IL to the nucleus accumbens shell (NAcSh) suppresses the aversive impact of sucrose devalued using the conditioned taste aversion paradigm in males and female rats. In naive rats, no significant differences in appetitive or aversive taste reactivity (TR) to sucrose was observed indicating that initial sucrose palatability was equivalent across sex. However, we found that optical activation of the ILNAcSh pathway during intraoral infusion of devalued sucrose inhibited aversive TR in male but not female rats. Interestingly, when allowed to freely ingest water and sucrose in a two-bottle test both males and females with a history of IL-NAcSh stimulation exhibited greater preference for sucrose. Optical pathway activation failed to alter TR to innately bitter quinine in either sex. Finally, both sexes lever pressed to self-stimulate the IL-NAcSh pathway. These results indicate that the IL-NAcSh pathway plays an important role in suppressing learned aversive states selectively in males but spares hedonic processing of innately aversive tastants. Further, pathway activation is reinforcing in both sexes, indicating that suppression of conditioned aversive TR can be dissociable from the effects of unconditioned rewarding properties of IL-NAcSh pathway activation.
\end{abstract}

Key words: affect; behavior; emotion; hedonics; infralimbic; shell

Significance Statement

Negative emotional states contribute to psychiatric disorders including depression and substance use disorders. In this study, we examined whether brain circuitry previously implicated in suppressing negative emotional states in humans can inhibit learned aversion in male and female rats. We found that optical activation of the infralimbic to nucleus accumbens shell pathway attenuates learned aversive responses in male but not female rats, indicating an important sex difference in the function of this brain pathway. Furthermore, we found that pathway stimulation was reinforcing in both sexes. Collectively, these findings support the role of the infralimbic cortex and its projection to the nucleus accumbens shell in suppressing learned negative emotional states and highlight an important sex-specific function of this pathway.

\section{Introduction}

The hedonic properties of stimuli profoundly impact behavior (Young, 1952; Kringelbach and Berridge, 2017), and psychiatric disorders are often associated with disrupted hedonic processing

Received Jan. 17, 2020; revised July 14, 2020; accepted July 27, 2020.

Author contributions: S.W.H. designed research; S.W.H. performed research; S.W.H. analyzed data; R.M.C. wrote the paper.

The authors declare no competing financial interests.

This work was supported by National Institutes of Health Grant DA-014339 to R.M.C. and 1F2-MH-115653-

$01 A 1$ to S.W.H.). We thank Xuefei Wang and Joey Sloand for outstanding technical support.

Correspondence should be addressed to Regina M. Carelli at rcarelli@unc.edu.

https://doi.org/10.1523/JNEUROSCI.0137-20.2020

Copyright $\odot 2020$ the authors
(Der-Avakian and Markou, 2012; Kringelbach and Berridge, 2017). For example, individuals experiencing depression exhibit anhedonia, or the loss of pleasure, and heightened levels of dysphoria (Pizzagalli et al., 2008; Der-Avakian and Markou, 2012). As such, it is important to develop a better understanding of the neural systems that regulate normal hedonic processing and suppress negative emotional states to provide a foundation for the development of treatments that can target these systems to alleviate negative affect in psychiatric illnesses.

Taste reactivity (TR) provides a preclinical approach to study hedonic processing. In the TR model, tastants are infused directly into the mouths of rats, and in response they exhibit a set of behaviors that reflect the hedonic value of the tastant. For 


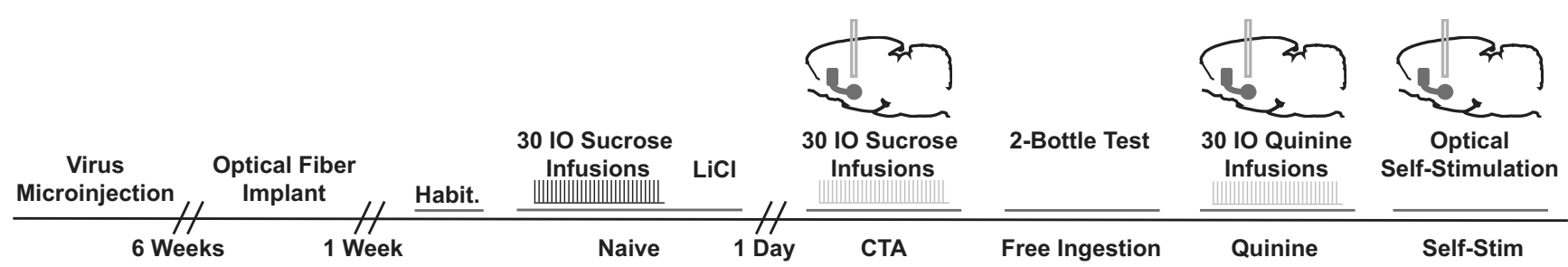

Figure 1. Experiment timeline. Rats received virus microinjections and were allowed 6 weeks for virus expression in IL cell bodies and projections to the NAcSh. Optical fibers and I0 catheters were implanted, and rats were allowed to recover, then habituated to 10 infusion. In the naive state, rats received I0 infusions of sucrose and TR was recorded. Immediately thereafter, rats received an injection of $\mathrm{LiCl}$ to create an association between sucrose and illness then given a $1 \mathrm{~d}$ break before CTA testing. However, each 10 sucrose infusion in the CTA test was paired with optical activation of the IL-NAcSh pathway and TR was measured. The next day rats could freely ingest water and sucrose (two-bottle test) in their homecage for $2 \mathrm{~h}$. A day later, rats received 10 infusion of quinine along with optogenetic stimulation of the IL-NAcSh pathway to determine whether pathway activation impacts TR to an innately aversive tastant. Finally, rats could selfstimulate the IL-NACSh pathway for $3 d$.

example, sweet sucrose elicits appetitive TR (e.g., licking), while bitter quinine evokes aversive TR, such as gaping. In the conditioned taste aversion (CTA) model, rats will devalue sweet solutions after they are paired with malaise-inducing lithium chloride ( $\mathrm{LiCl})$. Naive rats display appetitive TR to sucrose, but after pairing the sweet with $\mathrm{LiCl}$, sucrose comes to evoke aversive TR. As such, CTA provides an approach to understand the neural mechanisms governing learned changes in hedonic value. Importantly, the basic mechanisms of reward and aversion are translationally relevant as they are thought to contribute to complex emotional states in humans (Eisenberger et al., 2003; Kringelbach and Berridge, 2017).

The human ventromedial prefrontal cortex (vmPFC) has an important role in affect regulation and extinction learning (Eisenberger et al., 2003; Phan et al., 2005; Johansen-Berg et al., 2008; Delgado et al., 2016). The rat homolog of the vmPFC is the infralimbic cortex (IL; Uylings et al., 2003; Heilbronner et al., 2016), and activation of the IL promotes extinction of learned aversive states, such as conditioned fear (Do-Monte et al., 2015) and CTA (Mickley et al., 2005; Akirav et al., 2006; Maroun et al., 2012). As such, the IL likely functions to suppress learned negative hedonic states such as fear and negative affect. Importantly, the IL is embedded within a larger neural circuit, and it remains unclear how the IL may impact other brain regions to suppress learned aversive states. In this regard, the IL sends a dense projection to the nucleus accumbens shell (NAcSh), a region that is heavily implicated in affective processing (Sesack et al., 1989; Takagishi and Chiba, 1991; Reynolds and Berridge, 2002; Vertes, 2004; Roitman et al., 2005, 2010), and the IL-NAcSh circuit likely contributes to learned aversion.

Here, we determined whether activation of the IL-NAcSh pathway using optogenetics would suppress innate and conditioned aversive TR. Further, sex differences in IL neuronal morphology and function have been noted by others (Wang et al., 2009; Farrell et al., 2013), raising the possibility that the IL has distinct functions that are unique to males compared with females. As such, we also evaluated whether males and females exhibited different behavioral responses to optical pathway activation. We found that optical activation of the IL-NAcSh pathway inhibited learned aversive hedonic reactions to devalued sucrose in male rats, but not female rats. However, optical pathway stimulation did not alter TR responses to innately aversive quinine in either sex. When allowed to freely ingest water and sucrose, a history of pathway activation promoted sucrose preference in both male and female rats. Finally, we found that both sexes lever-pressed to self-stimulate the IL-NAcSh pathway. Collectively, these results indicate that activation of the ILNAcSh pathway potently suppresses learned aversive hedonic reactions in male rats but not in females; a finding that suggests important physiological distinctions between hedonic neural circuitry across sexes. Further, pathway activation enhanced sucrose preference in a two-bottle test and was reinforcing (elicited self-stimulation) in males and females, demonstrating common effects of pathway activation on innate hedonic processing across sexes.

\section{Materials and Methods}

Subjects. Male and female Sprague Dawley rats weighing between 220 and $350 \mathrm{~g}$ at the beginning of the study were used (Envigo). All rats were single housed and maintained on a $12 \mathrm{~h}$ reverse light/dark cycle (all testing occurred during the dark phase). Rats were provided ad libitum access to water and chow (Tekland 2920x, Envigo) except during all behavioral testing where water was restricted to $20-25 \mathrm{ml} / \mathrm{d}$. All protocols were conducted in accordance with the National Institutes of Health Guidelines for the Care and Use of Laboratory Animals and were approved by the University of North Carolina at Chapel Hill Institutional Animal Care and Use Committee.

Group assignment and surgeries. A $2 \times 2$ experimental design was used [mCherry vs Channelrhodopsin $(\mathrm{ChR} 2) \times$ male vs female]. Rats were divided into the following four groups: (1) male rats that received mCherry $(n=8)$; (2) male rats that received ChR2 $(n=7)$; (3) female rats that received mCherry $(n=7)$; and (4) female rats that received ChR2 $(n=11)$. Of note, some rats were euthanized before self-stimulation testing such that the number of self-stimulation animals were 7 mCherry males, 6 ChR2 males, 7 mCherry females, and 10 ChR2 females.

To induce virus expression in glutamatergic IL neurons, rats were anesthetized with a mixture of ketamine $(100 \mathrm{mg} / \mathrm{kg})$ and xylazine $(10 \mathrm{mg} /$ $\mathrm{kg}$, i.p.) and microinjected with $500 \mathrm{nl}$ of mCherry (AAV5-CamKIIamCherry; UNC Vector Core) or channelrhodopsin [AAV5-CamKIIahchR2(h134R)-mCherry] under the CaMKII promoter bilaterally in the IL (coordinates: anteroposterior, $+2.7 \mathrm{~mm}$; medial-lateral, $\pm 0.5 \mathrm{~mm}$; dorsal-ventral, $4.7 \mathrm{~mm}$ ) at a concentration of $3.2 \times 10^{12}$ viral genomes $/ \mathrm{ml}$. Virus was microinjected at a rate of $100 \mathrm{nl} / \mathrm{min}$, and injectors were left in place for $10 \mathrm{~min}$ postmicroinjection to ensure virus spread. Rats were allowed at least 6 weeks of recovery for virus to express in IL-NAcSh projections. A second surgery was performed to implant an intraoral catheter and optical fibers ( $200 \mu \mathrm{m}$ core) aimed at the medial rostral NAcSh (anteroposterior, $+1.5 \mathrm{~mm}$; medial-lateral, $\pm 2.1 \mathrm{~mm}$; dorsal-ventral, -6.5 $\mathrm{mm}$; from skull, lowered at a $10^{\circ}$ angle) using established procedures (Saddoris et al., 2015; Hurley et al., 2017). After each surgery, rats received subcutaneous injections of meloxicam ( $1 \mathrm{mg} / \mathrm{kg} / \mathrm{d}$ for $3 \mathrm{~d})$. Following the second surgery, rats also received subcutaneous enrofloxacin injections $(5 \mathrm{mg} / \mathrm{kg}, 2$ injections $/ \mathrm{d}$ for $5 \mathrm{~d}$ ) to prevent infection associated with intraoral catheter implant. An additional week of recovery occurred before testing began.

Behavioral testing. A timeline of behavioral testing is presented in Figure 1. After completion of surgeries and recovery, rats were habituated to the test chamber $(2 \mathrm{~d})$ and then mildly water restricted. Rats were then habituated to intraoral (IO) infusion (IO water delivered). Testing 
began the day after habituation to IO infusion, as indicated by robust licking in response to IO water. On day 1 of testing, naive rats received 303.5 -s-duration IO infusions of $0.5 \mathrm{~m}$ sucrose delivered on a variable interval $30 \mathrm{~s}$ schedule, and TR was recorded during each infusion. Immediately after infusions concluded, rats were injected with $\mathrm{LiCl}(127 \mathrm{mg} / \mathrm{kg}$, i. p., $0.3 \mathrm{M} \mathrm{LiCl}$ ) to induce CTA. Rats were allowed a 1 $\mathrm{d}$ break to allow for recovery from $\mathrm{LiCl}$ injection and then CTA testing began. During CTA testing, rats received IO sucrose as described above; however, optical illumination of the IL-NAcSh pathway ( $5 \mathrm{~s} \mathrm{du}-$ ration, $10 \mathrm{~mW} 473 \lambda$ laser at a frequency of $20 \mathrm{~Hz}$ ) coincided with each IO infusion in both ChR2 and mCherry rats (to control for a heating artifact), and TR was recorded. Stimulation parameters were chosen based on previous work indicating that IL optical stimulation at this frequency inhibits conditioned fear (Do-Monte et al., 2015). To determine whether optical illumination had lasting effects on sucrose preference, the next day rats could freely ingest water and $0.5 \mathrm{M}$ sucrose in their homecage for $2 \mathrm{~h}$ (optical illumination absent). The following day, rats received IO infusions of $1 \mathrm{~mm}$ quinine along with optical illumination, as described above, to determine whether activation of the IL-NAcSh pathway would impact TR to an innately aversive tastant. Finally, rats could self-stimulate the IL-NAcSh pathway for $3 \mathrm{~d}$ in 30 min sessions. During self-stimulation testing, a cuelight was present over an extended lever, and each lever press resulted in a $5 \mathrm{~s}$ tone accompanied by $5 \mathrm{~s}$ optical illumination using the parameters described above. A 15 s timeout occurred after laser illumination signaled by termination of the cue-light.

Taste reactivity. Taste reactivity was assessed as described previously (Grill and Norgren, 1978) by an experimenter blind to treatment conditions. Lateral tongue protrusions, rhythmic tongue protrusions, and paw licking were scored as appetitive TR. Behaviors that actively removed tastant from the mouth such as gaping, mouth-to-floor wipes, and mouth wipes were scored as aversive. Passive drips (PDs), where fluid drips passively from the mouth instead of being actively removed, were quantified separately as done by others (Parker et al., 1992). TR is presented as the total number of appetitive, aversive, and PD responses.

Histology. At the end of testing, rats were heavily anesthetized with a mixture of ketamine $(100 \mathrm{mg} / \mathrm{kg})$ and xylazine $(10 \mathrm{mg} / \mathrm{kg}$, i.p.) and perfused transcardially with PBS followed by $4 \%$ paraformaldehyde (Millipore Sigma). Brains were collected, postfixed in $4 \%$ paraformaldehyde for $24 \mathrm{~h}$ followed by $20 \%$ sucrose in $0.1 \mathrm{~m}$ phosphate buffer, and then sectioned. Sections were mounted on slides and imaged with a Leica DFC $45^{\circ} \mathrm{C}$ wide-field microscope or a Zeiss LSM 800 confocal microscope. IL-NAcSh pathway virus expression was verified, and optical fiber placements were recorded along the rostral-caudal NAcSh axis based on optical fiber tracks. Rostral-caudal optical fiber location was identified and mapped onto an atlas (Paxinos and Watson, 2007). We determined the extent to which each pair of optical fiber placements impacted aversive TR during the CTA test by comparing aversive TR from each ChR2 rat to average aversive TR of same-sex mCherry controls [(aversive TR/average aversive TR of same-sex controls) $\times 100]$.

Statistics. For all taste reactivity and fluid consumption analyses, group differences were analyzed using two-way repeated-measures ANOVAs followed by Newman-Keul's post-tests. A three-way ANOVA was used to examine differences in self-stimulation. All analyses were conducted in GraphPad Prism version 8.1.2.

\section{Results}

\section{Histology}

Virus expression was validated in the IL with projections in the NAcSh, illustrated in Figure 2. An example of virus spread at IL injection sites is overlaid on an atlas plate (Fig. 2A). Expression of neuronal projections and an optical fiber in the NAcSh (NAcSh delineated by solid line) is presented in Figure $2 B$. Confocal images show neuronal cell body virus expression in the IL (Fig. 2C) and projections in the NAcSh (Fig. 2D). We observed ChR2 and mCherry expression in IL cell bodies and NAcSh projections in both males and females. Further, we verified that the distribution of IL projections to the limbic forebrain were consistent with prior IL anterograde tracing findings (Vertes, 2004). Rats with missed virus microinjections were removed from analysis. Optical fibers from animals included in this study were in the rostral NAcSh, which has been linked to reward processing (Reynolds and Berridge, 2002; +2.52 to +1.92 AP according to Paxinos and Watson, 2007). Two male rats had optical fibers located in the caudal pole of the caudal shell (anteroposterior, +1.08 ), which has been linked to aversion processing (Reynolds and Berridge, 2002), and these rats were removed from analysis.

Activation of the IL-NAcSh pathway suppressed conditioned aversive responses in male but not female rats

Naive rats received IO sucrose to ensure that TR was similar across all groups. No significant differences in appetitive or aversive TR to sucrose in naive rats were found (all $p$ values $>0.18$; Fig. $3 A, B$ ), indicating that initial sucrose palatability was equivalent across sex.

After sucrose- $\mathrm{LiCl}$ pairing, rats received IO sucrose concomitant with optical illumination and TR was measured. Analyses revealed a main effect of $\operatorname{sex}\left(F_{(1,29)}=14.32, p<0.001\right)$, no main effect of virus $\left(F_{(1,29)}=3.014, p=0.09\right)$, but a significant sex by virus interaction $\left(F_{(1,29)}=6.494, p<0.05\right)$. post hoc tests found that male ChR2 rats exhibited significantly less aversive TR compared with all other groups (Fig. $4 A, B ; p<0.01$ ). However, no effects were observed on appetitive TR (Fig. $4 C$; all $p$ values $>0.28$ ). Furthermore, no differences were observed in passive drips 
A

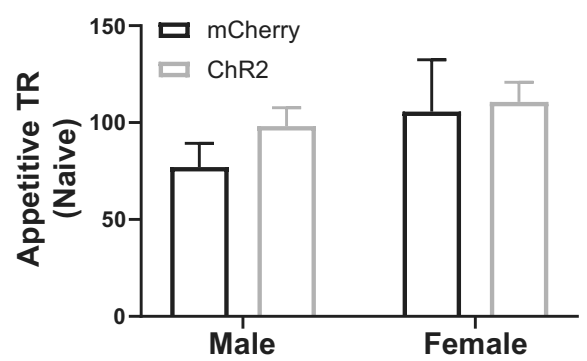

B

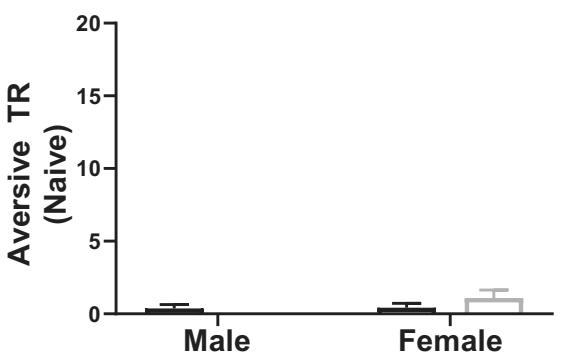

Figure 3. $\boldsymbol{A}, \boldsymbol{B}$, Appetitive $(\boldsymbol{A})$ and aversive $(\boldsymbol{B})$ TR to sucrose in naive rats. All groups exhibited similar levels of TR in the naive state (before (TA).

A

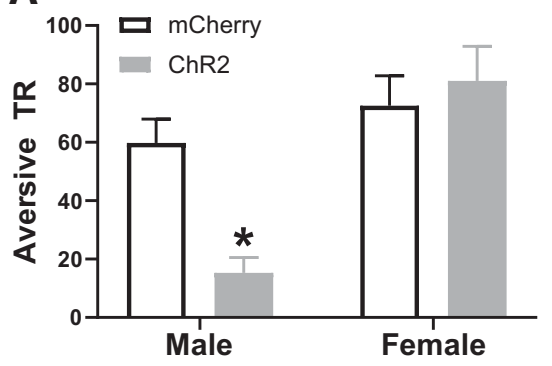

C

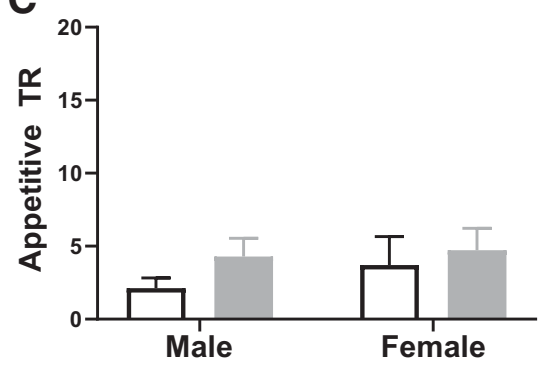

B

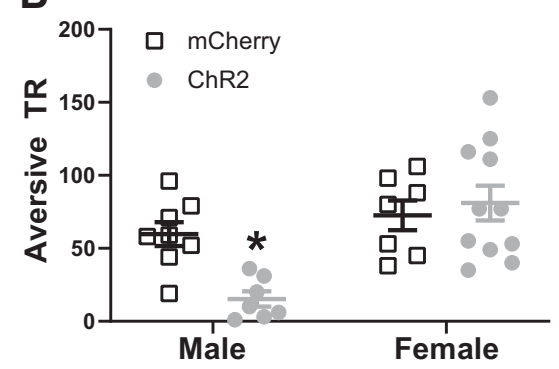

D

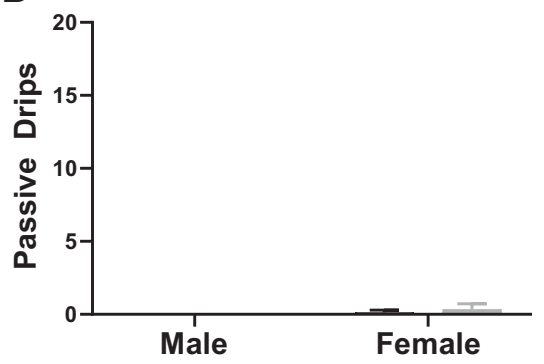

Figure 4. $\boldsymbol{A}-\boldsymbol{D}$, Bar graphs displaying mean aversive TR $(\boldsymbol{A})$, a scatterplot presenting individual aversive TR data points $(\boldsymbol{B})$, appetitive TR $(\boldsymbol{C})$, and passive drips $(\boldsymbol{D})$ to sucrose during (TA testing. Male rats that received optogenetic activation of the ILNACSh pathway showed a robust suppression of aversive TR ( ${ }^{*} p<0.01$ vs all other groups), but no change in appetitive TR or passive drips.

across groups, which occurred at negligible levels during CTA testing (Fig. $4 D$; all $p$ values $>0.33$ ). To ensure that differences in TR were not because of dissimilar rostral-caudal placements of optical fibers between sexes, we mapped optical fiber placements across the rostral-caudal axis by their ability to alter aversive TR (expressed as percentage of controls; Fig. 5). No clear effect of optical fiber placement was observed between sexes. These results show that stimulation of the IL-rostral NAcSh pathway attenuates conditioned aversive TR only in male rats without altering appetitive TR, indicating an important role for this pathway in suppressing learned aversion and a critical sex difference in the function of this pathway.

\section{Prior IL-NAcSh pathway activation enhanced sucrose preference}

The day after CTA testing, rats could freely ingest water and sucrose in a two-bottle test conducted in their homecage for $2 \mathrm{~h}$. Sucrose preference was calculated as (sucrose consumed - water consumed)/(sucrose consumed + water consumed) to yield a score from -1 to +1 that reflects sucrose preference. That is, a score of -1 indicates that rats only drank water while a score of +1 indicates that rats solely consumed sucrose. A two-way ANOVA revealed a significant main effect of virus $\left(F_{(1,29)}=7.735, p<0.01\right)$, but no effect of $\operatorname{sex}\left(F_{(1,29)}=0.04,227, p>0.83\right)$ or significant interaction $\left(F_{(1,29)}=1.039, p>0.31\right)$. These results indicate that a history of pathway stimulation during tastant infusion enhanced later sucrose preference in both sexes (Fig. 6A). Indeed, when collapsed across sexes an increase in sucrose preference was clear (Fig. 6B). We also analyzed total water and sucrose consumed to determine whether prior pathway stimulation had a broad impact on thirst or satiety and found no significant differences in total fluid consumption (all $p$ values $>0.49$; Fig. $6 C$ ). Together, these data reveal that a history of optical stimulation during tastant infusion enhanced subsequent sucrose preference in a twobottle test in both male and female rats, while total fluid intake was not altered, suggesting that unlearned homeostatic factors such as thirst and satiety were not influenced by prior stimulation.

\section{IL-NAcSh activation did not impact TR to innately aversive quinine} Following free fluid ingestion in the twobottle test, we examined whether activation of the IL-NAcSh pathway impacts aversive TR to innately aversive quinine. Analyses failed to find any significant effects of pathway stimulation on aversive TR to quinine (Fig. 7A; effects of sex:; $F_{(1,29)}=3.169, p>0.08$; virus: $F_{(1,29)}=$ $1.409, p>0.24$; interaction: $F_{(1,29)}=$ $0.5940, p>0.44)$. Further, we found no significant differences in appetitive TR, which occurred to a minor degree, across groups (Fig. $7 B$; all $p$ values $>0.35$ ). Finally, no differences were observed on passive drips across groups (Fig. 7C; effects of sex: $F_{(1,29)}=0.6448 p>0.42$; virus: $F_{(1,29)}=2.938$, $p>0.09$; interaction: $\left.F_{(1,29)}=0.0477, p>0.82\right)$. Together, these results indicate that pathway activation had no effect on TR to innately aversive quinine.

Male and female rats Self-Stimulate the IL-NAcSh pathway We examined whether rats would self-stimulate the IL-NAcSh pathway (Fig. 8). A three-way ANOVA revealed a main effect of virus $\left(F_{(1,26)}=8.174, p<0.01\right)$, but no significant effects of sex $\left(F_{(1,26)}=2.908 p>0.1\right)$, self-stimulation session $\left(F_{(1.393,36.21)}=1.768\right.$, $p>0.19]$, or any interactions (all $p$ values $>0.43$; Fig. $8 A$ ). While female rats appeared to self-stimulate less than males, this difference was not significant, and therefore these data indicate that both male and female rats expressing ChR2 self-stimulate the IL-NAcSh pathway. As there were no significant sex differences, self-stimulation data were collapsed across sex and presented in Figure $8 B$. 


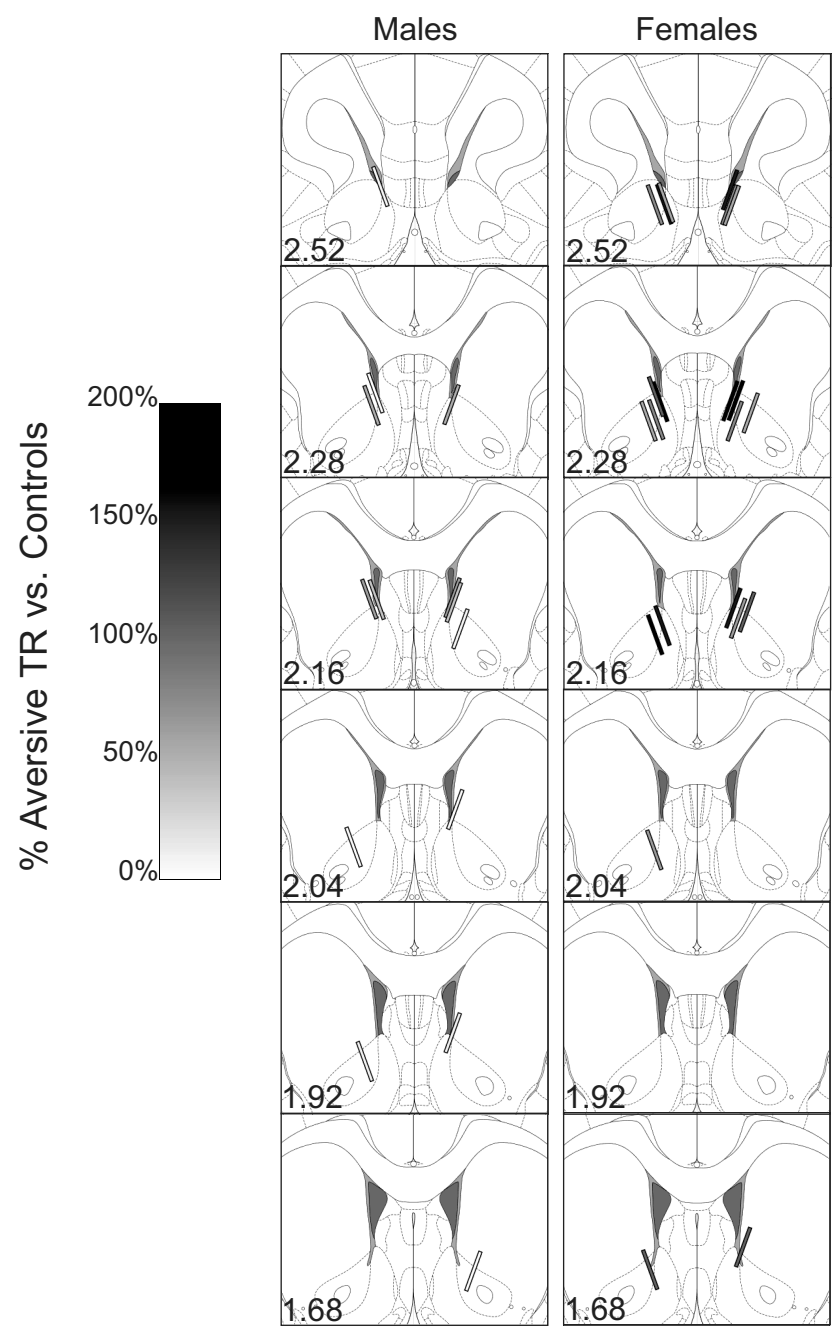

Figure 5. Optical fiber placements and their relationship to the suppression of learned aversive TR in males (left) and females (right). Coordinates according to Paxinos and Watson (2007) are displayed in the bottom left of coronal images. Optical fibers were primarily located in the rostral NACSh and are displayed as rectangles coded from light to dark based on the percentage change in aversive TR on the CTA test day compared with same-sex controls. Light shade indicates that aversive TR was suppressed, while dark shade indicates that aversion was enhanced.

\section{Discussion}

We investigated the role of the glutamatergic IL-NAcSh pathway in suppressing conditioned (learned) and innate aversion in male and female rats. We found that optical activation of the IL-NAcSh pathway inhibited conditioned aversive responses in male, but not female, rats, while sparing innate aversive responses to quinine. Further, male and female rats with a history of pathway stimulation exhibited greater sucrose preference when administered two-bottle tests the next day in their homecages. Finally, male and female rats self-stimulated the pathway, supporting prior research indicating that IL-NAcSh pathway stimulation is reinforcing (Britt et al., 2012). Together, we found sex-specific effects of IL-NAcSh pathway activation on suppression of learned aversion in males but not females, but also common effects of pathway activation on enhanced sucrose preference and reinforcement across sexes. Importantly, our findings support a distinction between the inhibition of conditioned negative hedonic states and the unconditioned rewarding properties of IL-NAcSh pathway activation.
A major finding of the present study is that optical activation of the glutamatergic IL-NAcSh pathway suppressed conditioned aversive TR in male but not female rats. Further, optical pathway stimulation had no effect on appetitive TR in either sex, demonstrating that pathway activation did not alter the hedonic valence of devalued sucrose by enhancing palatability. As such, our findings indicate that a male-specific function of the IL-NAcSh pathway is to suppress learned aversive TR without imparting positive hedonic value to a devalued tastant. This finding is congruent with other reports that found IL activation inhibits aversive states including conditioned and pharmacologically induced fear, as well as CTA (Maroun et al., 2012; Richard and Berridge, 2013; Do-Monte et al., 2015). Our results expand on these findings by showing that the IL-NAcSh pathway suppresses the aversive component of CTA in males. It is unlikely that basic sex differences in CTA learning mediate the present findings, as aversion in mCherry rats was similar across sexes (Fig. 4A,B). Further, prior studies found females exhibit rapid extinction of CTA compared with males, indicating that the aversion memory is more labile in females (Chambers and Sengstake, 1976). Finally, optical pathway activation had no effects on TR to innately aversive quinine in males or females, suggesting that the IL-NAcSh pathway specifically functions to suppress learned aversive states in males.

A functional heterogeneity in reward and aversion processing exists along the rostral-caudal NAcSh. The rostral NAcSh is associated with positive hedonic states and appetitive motivated behaviors, while the causal shell is linked to aversion (Reynolds and Berridge, 2002; Faure et al., 2010; Richard and Berridge, 2013; Castro and Berridge, 2014; Hurley et al., 2017). Therefore, the IL projection to the rostral NAcSh was targeted in the present experiment based on its role in driving appetitive motivated behavior. Furthermore, prior research found that IL activation suppresses both appetitive eating behavior and aversive defensetreading behaviors evoked by glutamate antagonism in the rostral (evoked eating behavior) and caudal shell (evoked defensive treading; Richard and Berridge, 2013), indicating that the IL plays a general role in inhibiting hedonically positive and negative behaviors within these NAc regions. We expand on those findings by showing that IL inputs to the rostral NAcSh suppress conditioned aversive TR. However, additional experiments testing whether IL-NAcSh stimulation alters responses to rewarding tastants are necessary to characterize the role of the IL-NAcSh pathway in hedonic processing. Further, activation of IL inputs to rostral versus caudal shell may differentially suppress hedonically positive and negative behaviors, but future work is needed to test this hypothesis.

Our results also support a growing literature on sex differences in IL function and neuron morphology. That is, others have shown sex differences in medial prefrontal cortex neuron morphology and sex-specific relationships between IL neuron morphology and extinction of freezing behavior (Farrell et al., 2013; Gruene et al., 2015). The findings are also consistent with human research showing that greater activation of the vmPFC is associated with extinction recall in men after undergoing conditioned fear learning and extinction, indicating the vmPFC may play a more important role in suppressing learned aversive states in men (Lebron-Milad et al., 2012).

When allowed to freely consume water and sucrose, both male and female rats with prior IL-NAcSh activation exhibited a greater preference for sucrose. Further, total fluid intake was not altered, indicating that homeostatic factors that influence intake such as thirst and satiety were not impacted by prior pathway 

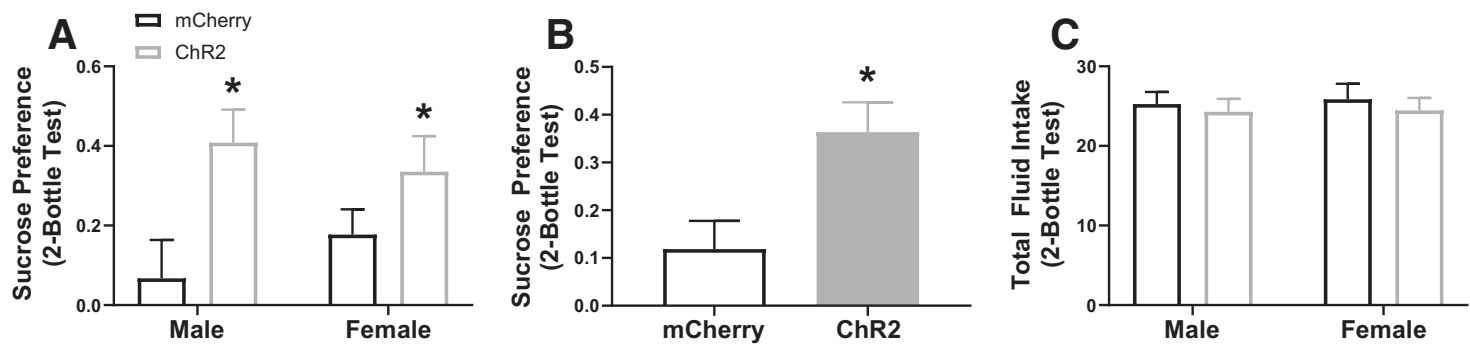

Figure 6. Taste reactivity during two-bottle test. $\boldsymbol{A}, \boldsymbol{B}$, Male and female rats that received prior optogenetic stimulation of the IL-NAcSh pathway during 10 sucrose infusion exhibited elevated preference for sucrose compared with mCherry controls $\left(\boldsymbol{A} ;{ }^{*} p<0.01\right.$ vs mCherry), also evident when data are collapsed across sex $\left(\boldsymbol{B} ;{ }^{*} p<0.01\right)$. $\boldsymbol{C}$, total fluid intake was equivalent across groups.

A

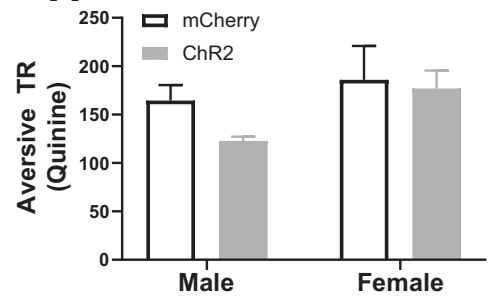

B

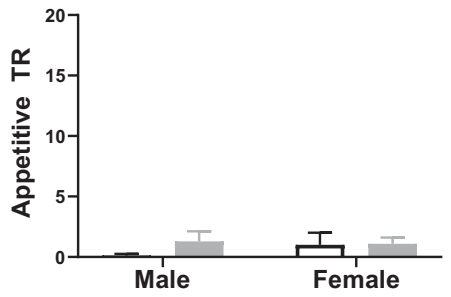

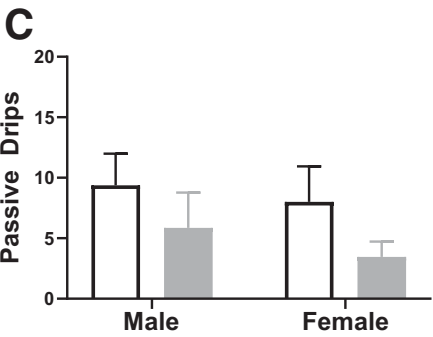

Figure 7. $\boldsymbol{A}-\boldsymbol{C}$, Aversive TR $(\boldsymbol{A})$, appetitive TR $(\boldsymbol{B})$, and passive drips $(\boldsymbol{C})$ to quinine infusions. No group differences were observed on TR to innately aversive quinine.
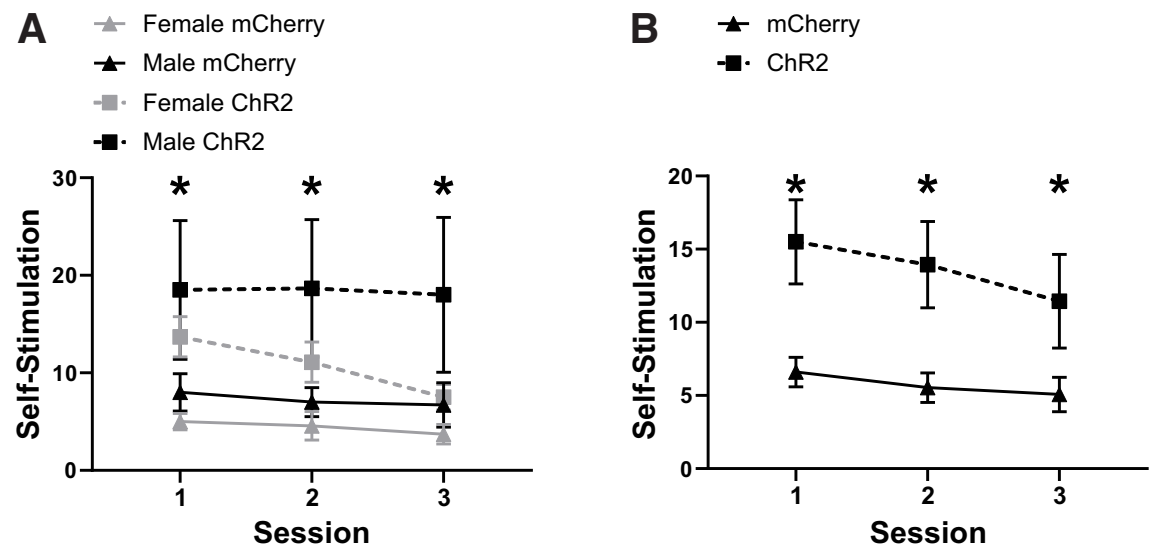

Figure 8. Lever press responses for optical IL-NAcSh optical activation presented over three self-stimulation sessions. $\boldsymbol{A}$, ChR2 rats self-stimulated more than mCherry controls independent of sex ( ${ }^{*} p<0.01$ vs mCherry rats). $\boldsymbol{B}$, Data collapsed across sex are presented $\left({ }^{*} p<0.05\right.$ vs mCherry).

activation. It is possible that pathway activation during infusions of devalued sucrose, which also elicited self-stimulation, promoted reward learning. That is, activation of the IL-NAcSh pathway may have conditioned a degree of reward to the devalued sucrose that ultimately contributed to enhanced sucrose preference in a two-bottle test. However, this possibility is not supported by our data showing that IL-NAcSh stimulation failed to elevate appetitive TR. An alternative possibility is that IL-NAcSh stimulation promoted extinction learning that ultimately elevated sucrose preference. Others have shown that high-frequency stimulation of the IL enhanced CTA extinction (Maroun et al., 2012). Similarly, optogenetic activation of the IL promotes extinction learning in the conditioned fear paradigm (Do-Monte et al., 2015), and the ILNAcSh pathway likely promotes extinction of cocaine self-administration (Warren et al., 2019). While extinction was not explicitly examined here, investigating the role of the IL-NAcSh pathway in extinction will likely be a fruitful avenue for future research.
The IL also projects to the lateral septum, medially adjacent to the NAcSh. One concern is whether some of the effects observed in the present study were because of lateral septum stimulation. However, preliminary data from male ChR2 rats that had one optical fiber in the lateral septum and a second fiber in the NAcSh indicated that ipsilateral lateral septum/rostral NAcSh stimulation failed to suppress learned aversion during CTA testing, although these rats did exhibit elevated sucrose preference. Furthermore, prior research indicates that ablating the septum tends to promote fluid consumption, suggesting an inhibitory role for the septum in fluid intake (Siegel, 1976; Vasudev et al., 1985; but see McGowan et al., 1972). Future studies are needed to more completely characterize the role of the lateral septum in these processes.

Both males and females lever pressed to self-stimulate the ILNAcSh pathway, indicating that the pathway is reinforcing and is consistent with prior findings (Britt et al., 2012). However, since pathway activation did not impact aversive TR in females, it is unlikely that the reinforcing effects of pathway activation were the underlying cause of reduced aversive TR observed in males. Nor did pathway activation elevate appetitive TR in either sex. Collectively, our findings indicate that a sex-specific divergence occurs between IL-NAcSh pathway activation effects on inhibiting conditioned aversive responses (only occurs in males), but pathway activation shares common effects on facilitating sucrose preference and inducing reinforcement.

Growing evidence indicates that the IL and human vmPFC play a critical role in regulating affective behavior. For example, 
the vmPFC is associated with suppressing negative emotions (Phan et al., 2005; Johansen-Berg et al., 2008), impulse control in boys (Boes et al., 2009), and inhibition of the stress response (Urry et al., 2006). Rat studies also show that the IL inhibits fear (Richard and Berridge, 2013; Do-Monte et al., 2015), eating (Richard and Berridge, 2013), impulsive responses (Dalley et al., 2011), and the pathologic motivated behavior of drug seeking (Peters et al., 2008; Gutman et al., 2017; Warren et al., 2019). Our results provide additional support for IL regulation of affective behavior, especially in males. Together, it appears that the IL plays a critical role in inhibiting both appetitive and aversive behaviors and associated affective processes to provide animals with a greater degree of control over behavior.

The human vmPFC has been implicated in affect regulation and suppressing aversive emotional states (Mayberg et al., 1999; Phelps et al., 2004; Phan et al., 2005; Johansen-Berg et al., 2008). Importantly, we found that activation of the IL-NAcSh pathway inhibited aversive TR reactions to devalued sucrose in male rats, a finding that is consistent with the role of the vmPFC in emotion regulation and suggests that the projection from the vmPFC to the NAcSh may be a critical physiological substrate for affect regulation, especially in men. A history of pathway stimulation enhanced sucrose preference in males and females, a finding that raises the possibility that strengthening this pathway could be beneficial in helping to extinguish learned negative affective states in both sexes, although more work is necessary to test this hypothesis. Of note, deep brain stimulation of a region of the vmPFC that projects to the NAc alleviated depression in humans (Johansen-Berg et al., 2008), possibly by strengthening the vmPFC-rostral NAcSh pathway. If the vmPFC-rostral NAcSh pathway is involved in suppressing negative emotional states, treatments that bolster the efficacy of this pathway may prove beneficial in helping alleviate learned negative emotional states such as CTA that occur from chemotherapy, phobias, and negative affect that arises in major depressive disorder. Our findings support the view that this pathway may be a critical component of a therapeutically relevant neural circuit involved in emotional control and resilience (Maier and Watkins, 2010).

\section{References}

Akirav I, Khatsrinov V, Vouimba RM, Merhav M, Ferreira G, Rosenblum K, Maroun M (2006) Extinction of conditioned taste aversion depends on functional protein synthesis but not on NMDA receptor activation in the ventromedial prefrontal cortex. Learn Mem 13:254-258.

Boes AD, Bechara A, Tranel D, Anderson SW, Richman L, Nopoulos P (2009) Right ventromedial prefrontal cortex: a neuroanatomical correlate of impulse control in boys. Soc Cogn Affect Neurosci 4:1-9.

Britt JP, Benaliouad F, McDevitt RA, Stuber GD, Wise RA, Bonci A (2012) Synaptic and behavioral profile of multiple glutamatergic inputs to the nucleus accumbens. Neuron 76:790-803.

Castro DC, Berridge KC (2014) Opioid hedonic hotspot in nucleus accumbens shell: mu, delta, and kappa maps for enhancement of sweetness "liking" and "wanting". J Neurosci 34:4239-4250.

Chambers KC, Sengstake CB (1976) Sexually dimorphic extinction of a conditioned taste aversion in rats. Anim Learn Behav 4:181-185.

Dalley JW, Everitt BJ, Robbins TW (2011) Impulsivity, compulsivity, and top-down cognitive control. Neuron 69:680-694.

Delgado MR, Beer JS, Fellows LK, Huettel SA, Platt ML, Quirk GJ, Schiller D (2016) Viewpoints: dialogues on the functional role of the ventromedial prefrontal cortex. Nat Neurosci 19:1545-1552.

Der-Avakian A, Markou A (2012) The neurobiology of anhedonia and other reward-related deficits. Trends Neurosci 35:68-77.

Do-Monte FH, Manzano-Nieves G, Quiñones-Laracuente K, Ramos-Medina L, Quirk GJ (2015) Revisiting the role of infralimbic cortex in fear extinction with optogenetics. J Neurosci 35:3607-3615.
Eisenberger NI, Lieberman MD, Williams KD (2003) Does rejection hurt? An fMRI study of social exclusion. Science 302:290-292.

Farrell MR, Sengelaub DR, Wellman CL (2013) Sex differences and chronic stress effects on the neural circuitry underlying fear conditioning and extinction. Physiol Behav 122:208-215.

Faure A, Richard JM, Berridge KC (2010) Desire and dread from the nucleus accumbens: cortical glutamate and subcortical GABA differentially generate motivation and hedonic impact in the rat. PLoS One 5:e11223.

Grill HJ, Norgren R (1978) The taste reactivity test. I. Mimetic responses to gustatory stimuli in neurologically normal rats. Brain Res 143:263-279.

Gruene TM, Roberts E, Thomas V, Ronzio A, Shansky RM (2015) Sex-specific neuroanatomical correlates of fear expression in prefrontal-amygdala circuits. Biol Psychiatry 78:186-193.

Gutman AL, Nett KE, Cosme CV, Worth WR, Gupta SC, Wemmie JA, LaLumiere RT (2017) Extinction of cocaine seeking requires a window of infralimbic pyramidal neuron activity after unreinforced lever presses. J Neurosci 37:6075-6086.

Heilbronner SR, Rodriguez-Romaguera J, Quirk GJ, Groenewegen HJ, Haber SN (2016) Circuit-based corticostriatal homologies between rat and primate. Biol Psychiatry 80:509-521.

Hurley SW, West EA, Carelli RM (2017) Opposing roles of rapid dopamine signaling across the rostral-caudal axis of the nucleus accumbens shell in drug-induced negative affect. Biol Psychiatry 82:839-846.

Johansen-Berg H, Gutman DA, Behrens TEJ, Matthews PM, Rushworth MFS, Katz E, Lozano AM, Mayberg HS (2008) Anatomical connectivity of the subgenual cingulate region targeted with deep brain stimulation for treatment-resistant depression. Cereb Cortex 18:1374-1383.

Kringelbach ML, Berridge KC (2017) The affective core of emotion: linking pleasure, subjective well-being, and optimal metastability in the brain. Emot Rev 9:191-199.

Lebron-Milad K, Abbs B, Milad MR, Linnman C, Rougemount-Bücking A, Zeidan MA, Holt DJ, Goldstein JM (2012) Sex differences in the neurobiology of fear conditioning and extinction: a preliminary fMRI study of shared sex differences with stress-arousal circuitry. Biol Mood Anxiety Disord 2:7.

Maier SF, Watkins LR (2010) Role of the medial prefrontal cortex in coping and resilience. Brain Res 1355:52-60.

Maroun M, Kavushansky A, Holmes A, Wellman C, Motanis H (2012) Enhanced extinction of aversive memories by high-frequency stimulation of the rat infralimbic cortex. PLoS One 7:e35853.

Mayberg HS, Liotti M, Brannan SK, McGinnis S, Mahurin RK, Jerabek PA, Silva JA, Tekell JL, Martin CC, Lancaster JL, Fox PT (1999) Reciprocal limbic-cortical function and negative mood: converging PET findings in depression and normal sadness. Am J Psychiatry 156:675-682.

McGowan BK, Hankins WG, Garcia J (1972) Limbic lesions and control of the internal and external environment. Behav Biol 7:841-852.

Mickley GA, Kenmuir CL, Yocom AM, Wellman JA, Biada JM (2005) A role for prefrontal cortex in the extinction of a conditioned taste aversion. Brain Res 1051:176-182.

Parker LA, Maier S, Rennie M, Crebolder J (1992) Morphine- and naltrexone-induced modification of palatability: analysis by the taste reactivity test. Behav Neurosci 106:999-1010.

Paxinos G, Watson C (2007) The rat brain in stereotaxic coordinates. London: Academic.

Peters J, LaLumiere RT, Kalivas PW (2008) Infralimbic prefrontal cortex is responsible for inhibiting cocaine seeking in extinguished rats. J Neurosci 28:6046-6053.

Phan KL, Fitzgerald DA, Nathan PJ, Moore GJ, Uhde TW, Tancer ME (2005) Neural substrates for voluntary suppression of negative affect: a functional magnetic resonance imaging study. Biol Psychiatry 57:210219.

Phelps EA, Delgado MR, Nearing KI, LeDoux JE (2004) Extinction learning in humans: role of the amygdala and vmPFC. Neuron 43:897-905.

Pizzagalli DA, Iosifescu D, Hallett LA, Ratner KG, Fava M (2008) Reduced hedonic capacity in major depressive disorder: evidence from a probabilistic reward task. J Psychiatr Res 43:76-87.

Reynolds SM, Berridge KC (2002) Positive and negative motivation in nucleus accumbens shell: bivalent rostrocaudal gradients for GABA-elicited eating, taste "liking"/"disliking" reactions, place preference/avoidance, and fear. J Neurosci 22:7308-7320. 
Richard JM, Berridge KC (2013) Prefrontal cortex modulates desire and dread generated by nucleus accumbens glutamate disruption. Biol Psychiatry 73:360-370.

Roitman MF, Wheeler RA, Carelli RM (2005) Nucleus accumbens neurons are innately tuned for rewarding and aversive taste stimuli, encode their predictors, and are linked to motor output. Neuron 45:587-597.

Roitman MF, Wheeler RA, Tiesinga PHE, Roitman JD, Carelli RM (2010) Hedonic and nucleus accumbens neural responses to a natural reward are regulated by aversive conditioning. Learn Mem 17:539-546.

Saddoris MP, Sugam JA, Stuber GD, Witten IB, Deisseroth K, Carelli RM (2015) Mesolimbic dopamine dynamically tracks, and is causally linked to, discrete aspects of value-based decision making. Biol Psychiatry 77:903-911.

Sesack SR, Deutch AY, Roth RH, Bunney BS (1989) Topographical organization of the efferent projections of the medial prefrontal cortex in the rat: an anterograde tract-tracing study with Phaseolus vulgaris leucoagglutinin. J Comp Neurol 290:213-242.

Siegel JL (1976) Effect of medial septal lesions on conditioned taste aversion in the rat. Physiol Behav 17:761-765.

Takagishi M, Chiba T (1991) Efferent projections of the infralimbic (area 25) region of the medial prefrontal cortex in the rat: an anterograde tracer PHA-L study. Brain Res 566:26-39.
Urry HL, van Reekum CM, Johnstone T, Kalin NH, Thurow ME, Schaefer HS, Jackson CA, Frye CJ, Greischar LL, Alexander AL, Davidson RJ (2006) Amygdala and ventromedial prefrontal cortex are inversely coupled during regulation of negative affect and predict the diurnal pattern of cortisol secretion among older adults. J Neurosci 26:4415-4425.

Uylings HB, Groenewegen HJ, Kolb B (2003) Do rats have a prefrontal cortex? Behav Brain Res 146:3-17.

Vasudev R, Gentil CG, Covian MR (1985) Taste preferences in a free-choice situation following electrical stimulation and lesion of septal area in rats. Physiol Behav 34:619-624.

Vertes RP (2004) Differential projections of the infralimbic and prelimbic cortex in the rat. Synapse 51:32-58.

Wang Z, Guo Y, Bradesi S, Labus JS, Maarek JI, Lee K, Winchester WJ, Mayer EA, Holschneider DP (2009) Sex differences in functional brain activation during noxious visceral stimulation in rats. Pain 145:120-128.

Warren BL, Kane L, Venniro M, Selvam P, Quintana-Feliciano R, Mendoza MP, Madangopal R, Komer L, Whitaker LR, Rubio FJ, Bossert JM, Caprioli D, Shaham Y, Hope BT (2019) Separate vmPFC ensembles control cocaine self-administration versus extinction in rats. J Neurosci 39:7394-7407.

Young PT (1952) The role of hedonic processes in the organization of behavior. Psychol Rev 59:249-262. 